\title{
Characterization of Silane Modified Polyvinyl Acetate Water Based Dispersion Film
}

\author{
Mindaugas Dubininkas ${ }^{1, *}$ and Gintaras Buika ${ }^{2}$ \\ ${ }^{1}$ SC "Achema", Jonalaukio k., Ruklos sen., LT-55550 Jonavos r., Lithuania \\ ${ }^{2}$ Department of Polymer Chemistry and Technology, Kaunas University of Technology, Radvilènu pl. 19, \\ LT-3028 Kaunas, Lithuania
}

\begin{abstract}
Major advantage of silane-modified systems is possibility to cross link reactive silanol groups and fortify polymer mechanical and chemical properties. However, application of modern analysis techniques and further characterization of macromolecular structure produced is complicated due to a poor solubility of silane modified polymer in various solvents. Silane modified polyvinyl acetate (PVAc) dispersions were produced by classic radical and RAFT polymerization using silane co-monomers and dispersion system stabilizer - silane modified industrial grade polyvinyl alcohol. The present paper deals with confirmation of silanol, siloxane bonds, silicon content and distribution, as well film surface morphology of silane modified PVAc dispersions films.
\end{abstract}

Keywords: Polyvinyl acetate, RAFT, alkoxysilane, siloxane bonds, element mapping.

\section{INTRODUCTION}

Polyvinyl acetate polymer and dispersion is well known products with certain parameters widely published in literature [1-3]. Nevertheless, applications of co-monomers, various dispersion system stabilizers change PVAc polymer end properties. Therefore, research of macromolecular structure is difficult and needs additional confirmations by using various analytical approaches. Despite the fact, production of silane modified PVAc in homogeneous and heterogeneous media was widely published in literature [4-10], poor solubility of polymer had a negative impact for determination of macromolecular structure and confirmation of silanol and/or siloxane bonds (Figure 1).

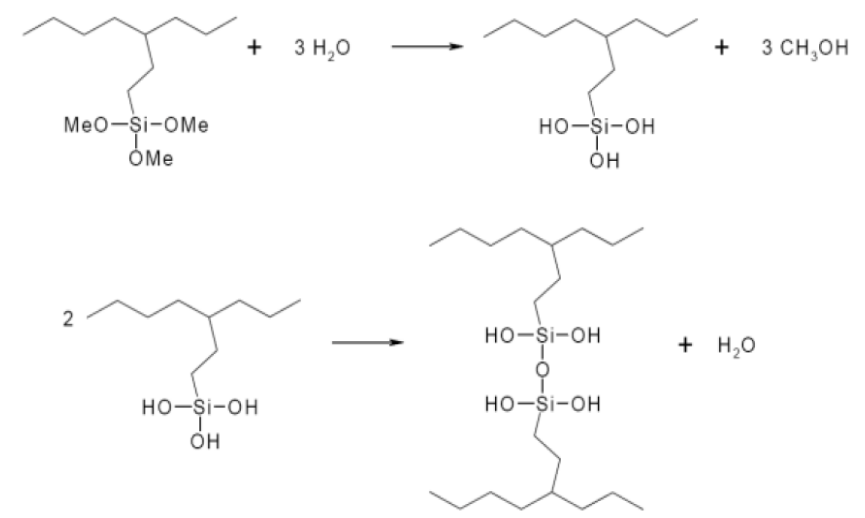

Figure 1: Hydrolysis and polycondensation of alkoxysilane side groups [11, 12].

*Address correspondence to this author at the SC "Achema", Jonalaukio k., Ruklos sen., LT-55550 Jonavos r., Lithuania; Tel: 00370-605-53711;

Fax: 00370-349-56378; E-mail: m.dubininkas@achema.com
Hydrolysis and condensation reactions of alkoxysilanes, described in Figure $\mathbf{1}$ are catalyzed both by acids and bases. In an acidic medium hydrolysis reactions undergo faster than condensation reactions, but in a basic medium condensation reactions are faster [13]. Industrial vinyl acetate emulsion polymerization proceeds in slightly acidic medium. Hydrolyzation of final dispersion is necessary in order to prevent PVAc hydrolyzation during storage. Accordingly, both alkoxysilane hydrolyzation and polycondensation reaction could proceed during and after alkoxysilane modified PVAc dispersion production. The present paper deals with confirmation of silanol, siloxane bonds in alkoxysilane modified PVAc dispersion films. For this purpose different dispersion production and analysis techniques were applied.

\section{EXPERIMENTAL AND ANALYSIS TECHNIQUES}

The samples for analysis were prepared from dispersions produced by using alkoxysilane (hereinafter silane) co-monomers with a brand name Geniosil GF31 and Geniosil GF51 (Wacker Silicones) and silane modified industrial grade PVA R1130 (Kuraray) as a dispersion system stabilizer [14, 15]. RAFT polymerization of vinyl acetate (VAc) was performed with 2-Cyanomethyl-N-methyl-Nphenyldithiocarbamate (Sigma Aldrich) according to a method for production of surfactant-stabilized dispersion published elsewhere [16]. The molar ratio of [RAFT/ammonium persulphate] $=0.5$, i.e. $[\mathrm{VAc} / \mathrm{RAFT}]$ $=977$. Experimental block scheme of silane modified PVAc dispersion production is presented in Figure $\mathbf{2}$. 


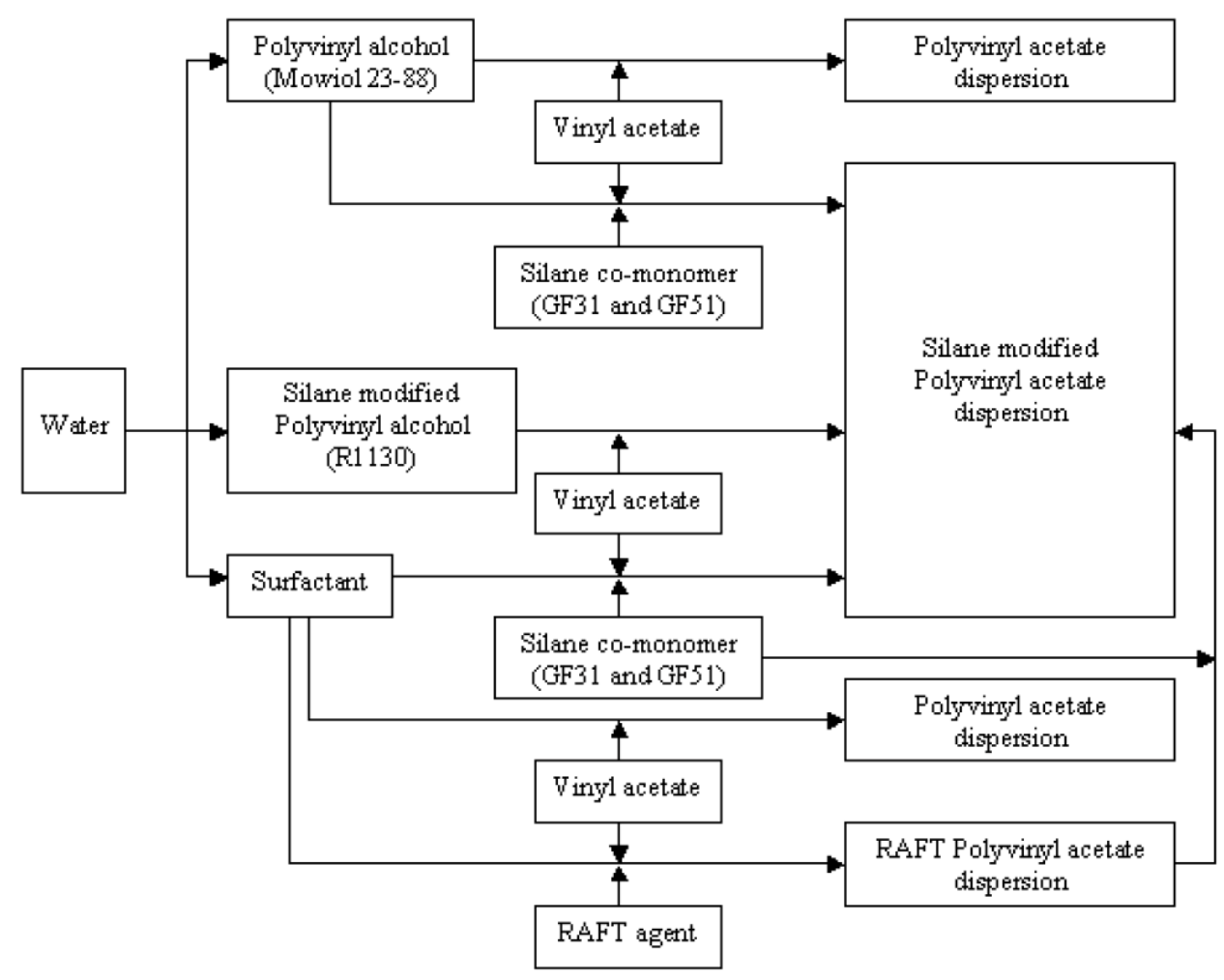

Figure 2: Silane modified polyvinyl acetate dispersion production routes.

Before analysis, dispersions were cast on a glass and dried at room temperature to a constant weight. Perkin-Elmer Pyris 1-TGA (PerkinElmer, USA) apparatus was used to study thermal decomposition of polymer. Thermal analyzer in nitrogen atmosphere at the heating rate of $10{ }^{\circ} \mathrm{C} / \mathrm{min}$. IR analysis was carried out using Spectrum GX FTIR (Perkin Elmer, USA), connected with ATR and "Spectrum 5.0.1" software. The samples - polymer films $(20 \times 50) \mathrm{mm}$. SEM analysis was performed using Quanta 200 FEG (FEI, USA) scanning microscope and X-Ray spectrometer Xflash 4030 (Bruker, Germany) was used for determination and mapping of silicon. Mass loss after polymer extraction was determined by washing polymer sample with toluene in Soxhlet extractor for 24 h. Extracted polymer was evaluated gravimetrically. In order to determine molecular weight differences among prepared polymers, samples were hydrolyzed with sodium hydroxide to PVA at boiling temperature for 1-2 $\mathrm{h}$ until full dissolution. Then, the polymer solution was cooled down, polymer was precipitated from water with acetone. Sodium hydroxide was washed from dried polymer by dissolution in demineralized water and further polymer precipitation with acetone. The procedure was repeated 3 times. After sodium hydroxide was washed out, polymer was dried to a constant mass, weighted and used for viscosity investigations. The viscosity-average molecular weight of polymers was calculated from intrinsic viscosity data measured with an Ubbelohde viscometer (Schott Duran, model 501 11/la). Analysis was carried out at $25^{\circ} \mathrm{C}$, demineralized water used as polymer solvent. Viscosimeter was calibrated against demineralized water, and the $K$ and $\alpha$ values were $20 \times 10^{-5} \mathrm{dL} / \mathrm{g}$ and 0.76 respectively [22]. Both $K$ and $\alpha$ are constants associated with Mark-Houwink-Sakurada equation, $[\eta]$ $=K M_{\vee}{ }^{\alpha}$, where $[\eta]$ is the intrinsic viscosity and $M_{v}$ the viscosity-average molecular weight of the polymer. Molecular weight measurement of THF soluble polymer fraction was performed by GPC using Malvern/Viscotek MSC system.

\section{RESULTS AND DISCUSSION}

Possible reactions between components and technological aspects should be considered understanding and comparing structure of unmodified and silane modified dispersion films. Silane incorporation could proceed during radical polymerization or polycondensation of silanols and PVAc $-\mathrm{OH}$ groups. It is possible to have silane or organosilanol and PVAc mixture at the end of dispersion production. Polymer structure changes could also proceed during dispersion drying - film formation. Hydrolyzation of silane side groups to silanols and further polycondensation could form cross- 
link macromolecular structures with siloxane bridges. In order to determine silane impact to PVAc dispersion film structure, the IR analysis of produced dispersion films was performed.

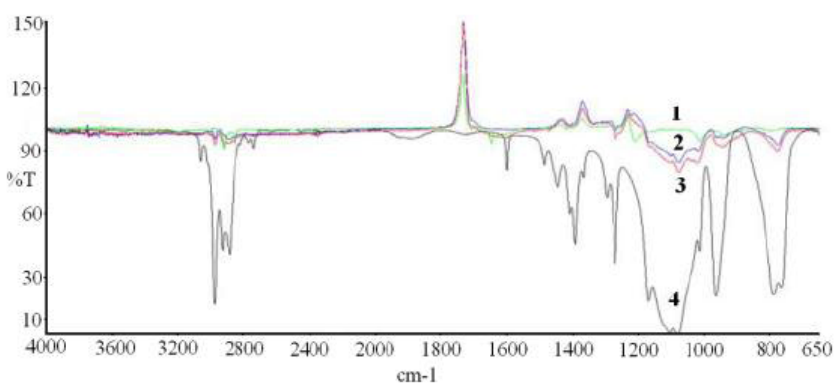

Figure 3: Difference IR spectra of PVA stabilized PVAc dispersion film, at VAc:GF51 weight ratio: 1 - 100:0.5 and 100:0; 2 - 100:1.5 and 100:0; 3 - 100:3 and 100:0. IR spectra. 4 - GF51.

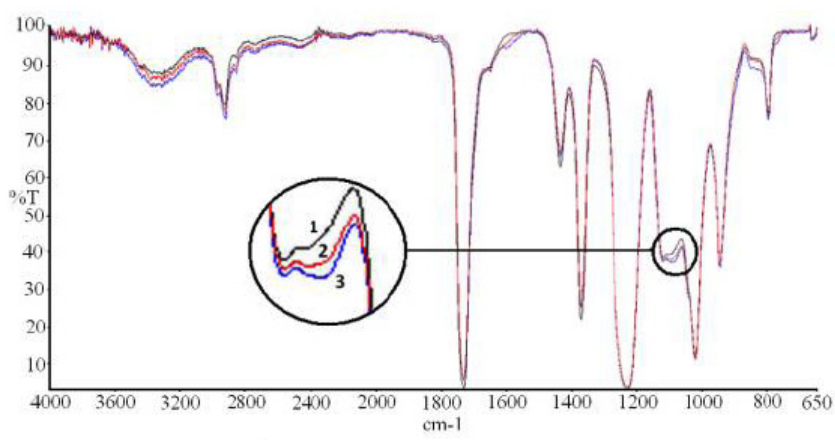

Figure 4: PVA stabilized PVAc dispersion film IR spectra, at VAc:GF31 weight ratio: 1 - 100:0; 2 - 100:0.5; 3 - 100:1.5.

According to GF51 silane IR spectra (Figure 3. 4), it can be seen absorption peaks at 1600; 1410; 1010; $960 \mathrm{~cm}^{-1}$, which could be attributed to $\mathrm{Si}-\mathrm{CH}=\mathrm{CH}_{2}$ group $\mathrm{C}=\mathrm{C}$ bond vibration. High intensity absorption peaks at $(1100-1075) \mathrm{cm}^{-1}$ could be attributed to $\mathrm{SiOCH} \mathrm{CH}_{3}$ group $\mathrm{Si}-\mathrm{O}$ bond vibrations. However absorption peaks at $(1100-1000) \mathrm{cm}^{-1}$ could be also attributed to $\mathrm{Si}-\mathrm{O}-\mathrm{Si}$ bond vibrations. The vibration peak at $1730 \mathrm{~cm}^{-1}$ could be attributed to PVAc acetate group $\mathrm{C}=\mathrm{O}$ bond vibrations. Vibration peak at $1647 \mathrm{~cm}^{-}$ ${ }^{1}$ could be attributed to VAc $C=C$ bond vibrations [17]. Low signal intensity of the peak shows low residual level of VAc in sample. Peaks at 3000, 2975 and 2800 $\mathrm{cm}^{-1}$ could be attributed to symmetric and asymmetric PVAc chain $\mathrm{C}-\mathrm{H}$ bond vibrations. IR spectra of GF51 modified samples showed different intensity of the absorption peaks at $(1100-1080) \mathrm{cm}^{-1}$ and $960 \mathrm{~cm}^{-1}$. The absorption peaks could be attributed to $\mathrm{Si}-\mathrm{O}-\mathrm{Si}$ bond vibrations $[18,19]$. Vibrations peak at $770 \mathrm{~cm}^{-1}$, could be attributed to $\mathrm{Si}-\mathrm{C}$ bond vibrations. The absence of $\mathrm{SiCH}=\mathrm{CH}_{2}$ group $\mathrm{C}=\mathrm{C}$ bond vibration peak at $1600 \mathrm{~cm}^{-1}$ and $\mathrm{VAc} C=\mathrm{C}$ bond vibration peak at 1651 $\mathrm{cm}^{-1}$ confirms copolymerization of VAc and GF51. High intensity absorption peak at $1720 \mathrm{~cm}^{-1}$, which could be attributed to $\mathrm{C}=\mathrm{O}$ bond vibrations, $\mathrm{COCH}_{3} \mathrm{CH}=\mathrm{CH}_{2}$ group $\mathrm{C}=\mathrm{C}$ bond vibration peak at $1638 \mathrm{~cm}^{-1}, \mathrm{SiOCH}_{3}$ group $\mathrm{C}-\mathrm{H}$ bond vibration peaks at 2946, $2842 \mathrm{~cm}^{-1}$ and $\mathrm{Si}-\mathrm{O}$ bond vibration peaks at $(1100-1080) \mathrm{cm}^{-1}$ determined in GF31 IR spectra [18, 19]. IR spectra of GF31 modified samples (Figure 4. 2, 3) are absence of VAc group $C=C$ bond vibration peak at $1638 \mathrm{~cm}^{-1}$ and characteristic for GF31 silane $\mathrm{SiOCH}_{3}$ group $\mathrm{C}-\mathrm{H}$ bond vibration peak at $2842 \mathrm{~cm}^{-1}$. Increase of VAc:GF31 weight ratio from $100: 0$ to $100: 1.5$, increased intensity of Si-O-Si bond vibration peak at $(1100-1080) \mathrm{cm}^{-1}$. In comparison to PVA stabilized PVAc dispersion, surfactant stabilized PVAc dispersion film IR spectra were absence of absorption peak at $3200 \mathrm{~cm}^{-1}$, which is characteristic for $\mathrm{O}-\mathrm{H}$ bond vibration.



Figure 5: IR spectra of film cast from dispersion produced by RAFT method, at VAc:GF51 weight ratio: 1 - 100:3; 2 difference between 100:0 and 1 .

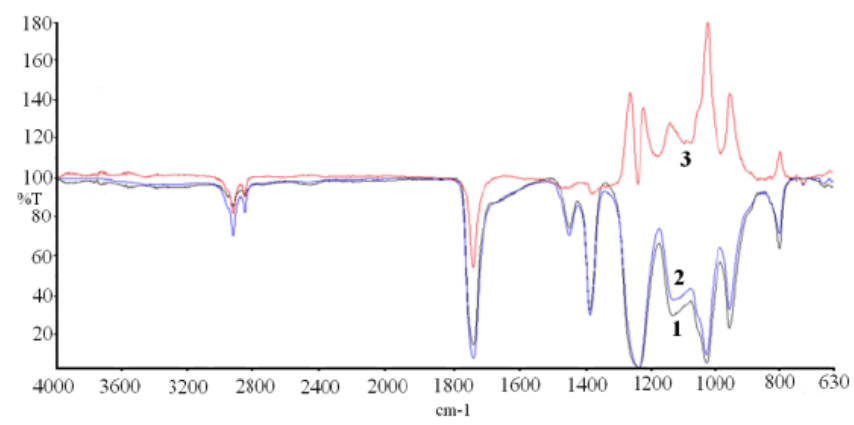

Figure 6: IR spectra of film cast from dispersion produced by RAFT method, at VAc:GF31 weight ratio: 1 - 100:0; 2 100:1; 3 - difference between 1 and 2.

The IR spectra of samples prepared from GF51 and GF31 modified PVAc dispersion produced by RAFT method is presented in Figures $\mathbf{5}$ and $\mathbf{6}$. According to IR spectra (Figure 5. 1), O-H bond vibrations peak at (3400-3200) $\mathrm{cm}^{-1}, \quad \mathrm{SiCH}=\mathrm{CH}_{2}$ group $\mathrm{C}=\mathrm{C}$ bond vibration peak $1600 \mathrm{~cm}^{-1}$ and $\mathrm{VAc} C=\mathrm{C}$ bond vibration 
Table 1: Weight Loss of PVA Stabilized Dispersion Films after Extraction with Toluene and Non-Volatile Matter Content at $500^{\circ} \mathrm{C}$. Intrinsic Viscosity [ $\eta$ ], Viscosity-Average Molecular Weight $\boldsymbol{M}_{\mathrm{v}}$ of Hydrolyzed Polymers

\begin{tabular}{|c|c|c|c|c|c|c|c|c|c|}
\hline Parameter & \multicolumn{9}{|c|}{ Dispersion film } \\
\hline Silane & - & \multicolumn{4}{|c|}{ GF51 } & \multicolumn{4}{|c|}{ GF31 } \\
\hline VAc:Silane weight ratio & 100:0 & $100: 0.5$ & $100: 1.5$ & $100: 3$ & $100: 6$ & 100:0.5 & $100: 1$ & $100: 1.5$ & $100: 3$ \\
\hline Weight loss, $\%$ & 78.6 & 38.3 & 13.8 & 7.9 & 3.4 & 11.5 & 9.1 & 7.7 & 1.5 \\
\hline$[\eta], \mathrm{dL} / \mathrm{g}$ & 0.17 & 0.24 & 0.30 & 0.31 & 0.32 & \multirow{2}{*}{\multicolumn{4}{|c|}{ Unhydrolyzable }} \\
\hline$M_{v} \times 10^{4}, \mathrm{~g} / \mathrm{mol}$ & 2.2 & 3.4 & 4.6 & 4.8 & 5.0 & & & & \\
\hline Non-volatile matter content, \% & 7.4 & - & 6.7 & - & 11.2 & - & 7.1 & - & 7.3 \\
\hline
\end{tabular}

peak at $1651 \mathrm{~cm}^{-1}$ were observed. Vibrations peak at (3400-3200) $\mathrm{cm}^{-1}$ (Figure 5. 1), could be attributed to silanol group $\mathrm{O}-\mathrm{H}$ bond vibrations, because of surfactant application instead of PVA. Absence of $\mathrm{O}-\mathrm{H}$ bond vibration peak in GF31 modified PVAc IR spectra (Figure 6. 1) confirm this presumption. Intense absorption peaks at (1220-1010) $\mathrm{cm}^{-1}$ and $960 \mathrm{~cm}^{-1}$ (Figure 5. 1) could be attributed to $\mathrm{Si}-\mathrm{O}-\mathrm{Si}$ bond vibrations [18, 19]. Regretfully, IR spectra of silane containing PVA (R1130) sample were absence of vibration peaks characteristic for silane modified samples. This can be determined by a small quantity of silane used in the manufacture of R1130.

Evaluation of branched macromolecules, linear polymers, oligomers were performed using sample extraction with toluene [20, 21]. Non-volatile matter content analysis was chosen for additional argumentation of silane participation in silane modified dispersion films. Following results are presented in Table 1

According to Table 1, weight loss of dispersion film after extraction greatly decreases by increase of VAc:GF51 or GF31 silane weight ratio applied in dispersion production. The difference between weight loss of unmodified and modified VAc:GF51 (100:6) and GF31 (100:3) samples reached 23 and 52 times, and it provides clear evidence of branched macromolecule polymer formation. Use small quantities of comonomers give negligible non-volatile matter content difference between unmodified and silane modified samples. Even though non-volatile matter content of sample with highest VAc:GF51 weight ratio was $3.8 \%$ higher in comparison to unmodified, presented results were in the range of analysis accuracy.

Partial solubility of silane modified and PVA stabilized PVAc polymers in various solvents cause additional analytical issues for researchers. To overcome solubility issue and measure molecular weight differences between unmodified and silane modified polymers, hydrolyzation of samples to water soluble form (PVA) was performed. GF51 modified samples were hydrolyzed successfully, however samples modified with GF31 silane were unhydrolyzable. According to results given in Table 1, viscosity-average molecular weight $M_{v}$ of hydrolyzed polymers increase at higher VAc:GF51 weight ratio. This could be explained by silane moiety incorporation in PVAc chain and/or formation of organosilanols. Surfactant stabilized PVAc dispersions were produced in order to exclude negative PVA impact to PVAc polymer solubility. Molecular weight of THF soluble polymer fraction was determined by GPC.

According to Figure 7 results, monomodal molecular weight distribution was determined for THF soluble
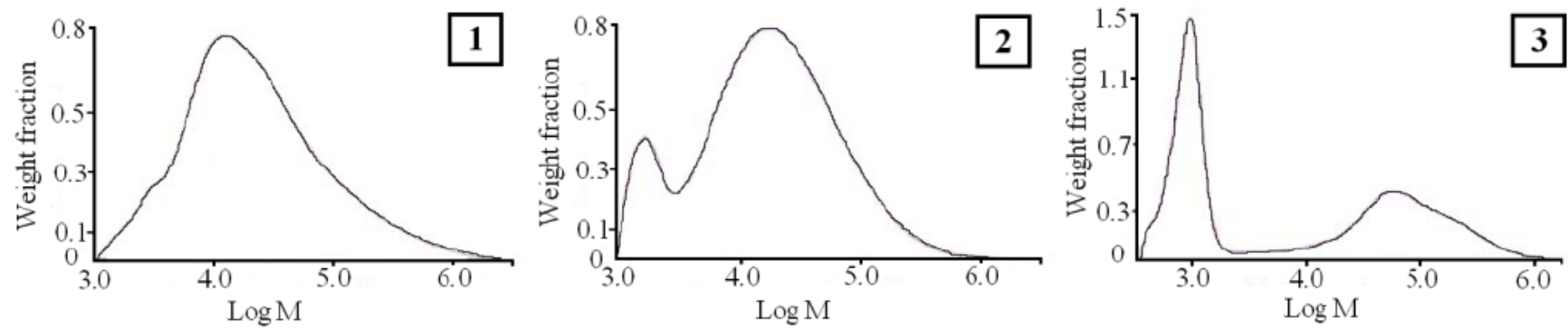

Figure 7: Molecular weight of THF soluble film fraction. Dispersions produced, at VAc:GF51 weight ratio: 1 - 100:0 by RAFT; 2 - 100:3; 3 - 100:3 by RAFT. 
Table 2: Silicon Content in Silane Modified PVAc Dispersion Films

\begin{tabular}{|c|c|c|c|c|c|}
\hline \multirow{2}{*}{ Silane } & \multirow{2}{*}{$\begin{array}{c}\text { VAc:Silane weight } \\
\text { ratio }\end{array}$} & \multirow{2}{*}{ System stabilizer } & \multicolumn{3}{|c|}{ Silicon } \\
\hline & & & $\%$ & Error, \% & Theoretical value, $\%$ \\
\hline GF51 & $100: 3$ & \multirow{2}{*}{ PVA } & 5.81 & 0.30 & 0.98 \\
\hline GF31 & $100: 1$ & & 0.55 & 0.10 & 0.18 \\
\hline \multirow{2}{*}{ GF51 } & $100: 3$ & \multirow{4}{*}{ Surfactant } & 0.96 & 0.07 & 0.92 \\
\hline & $100: 3$ (RAFT) & & 0.52 & 0.05 & 0.81 \\
\hline \multirow{2}{*}{ GF31 } & $100: 1$ & & 0.19 & 0.04 & 0.19 \\
\hline & 100:1 (RAFT) & & 0.51 & 0.05 & 0.15 \\
\hline
\end{tabular}

fraction of unmodified dispersion film 1. On the contrary, bimodal molecular weight distributions were determined for GF51 modified polymers 2 and 3. THF soluble fraction $(15-16) \times 10^{4}$ of unmodified film 1 could be attributed to PVAc. Therefore, THF soluble $5 \times 10^{4}$ molecular weight fraction determined in GF51 modified polymers 2 and 3 (produced by RAFT method) could be attributed to polycondensation products of oligosilanols.

Application of volatile silane co-monomers could determine silicon loss during dispersion production and/or dispersion drying. Therefore, additionally silicon content was determined by X-Ray. Following results of silicon content in dispersion films are presented in Table 2.

Theoretical values of silicon were calculated according to dispersion recipes published elsewhere $[14,16]$. According to Table 2 results, the minimum deviation between theoretical and analytical values were determined for films prepared from surfactant stabilized, GF51 and GF31 modified dispersions. Contrary to this, greatest difference was determined for PVA stabilized silane modified dispersion films: $5.8 \%$ and $0.98 \%$ (theoretical) at weight ratio VAc:GF51 $(100: 3)$ and $0.55 \%$ and $0.18 \%$ (theoretical) at VAc:GF31 (100:1). Whereas, analytical results were higher than theoretical, assumption regarding silane loss (evaporation) during production or film formation drying could be rejected. The main reason of such disagreement could be uneven silicon distribution in dispersion film. This is most likely, because difference between samples prepared from surfactant stabilized dispersions (except of RAFT) were much lower. Nevertheless, participation of silicon in silane modified dispersion films shows formation of polymerization products, not a simple silane (co-monomer) and PVAc dispersion mixture.

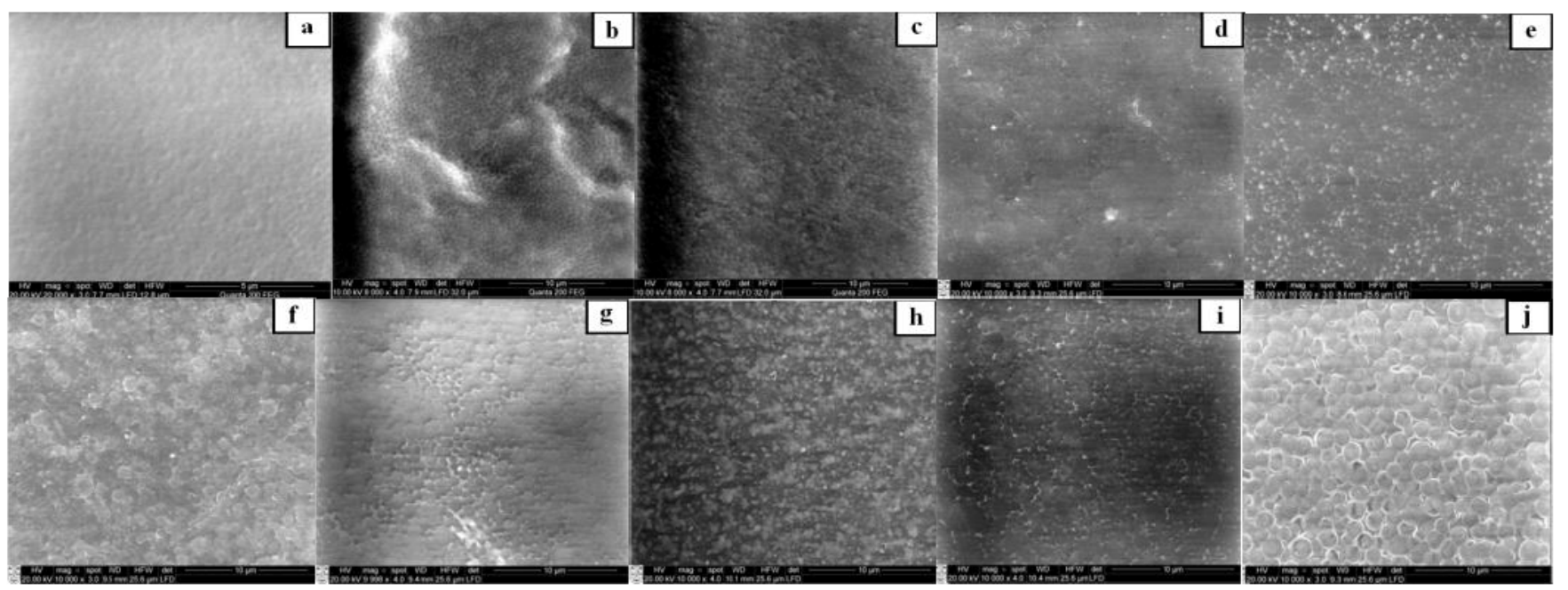

Figure 8: SEM micrographs of PVAc dispersions films. PVA stabilized, at VAc:GF51 weight ratio, magnification: a - 100:0, $\times 20000 ; \mathbf{b}-100: 3, \times 8000$. PVA stabilized at VAc:GF31 weight ratio, magnification: $\mathbf{c}-100: 1, \times 8000$. Surfactant stabilized, unmodified PVAc, magnification $\times 10000$ : d - PVAc; e - PVAc by RAFT. Surfactant stabilized at VAc:GF51 weight ratio 100:3: $\mathbf{f}$ - PVAc; g - PVAc by RAFT. Surfactant stabilized at VAc:GF31 weight ratio 100:1: h - PVAc; i PVAc by RAFT. Stabilized with silane modified PVA, magnification $\times 10000$ : j $-R 1130$. 

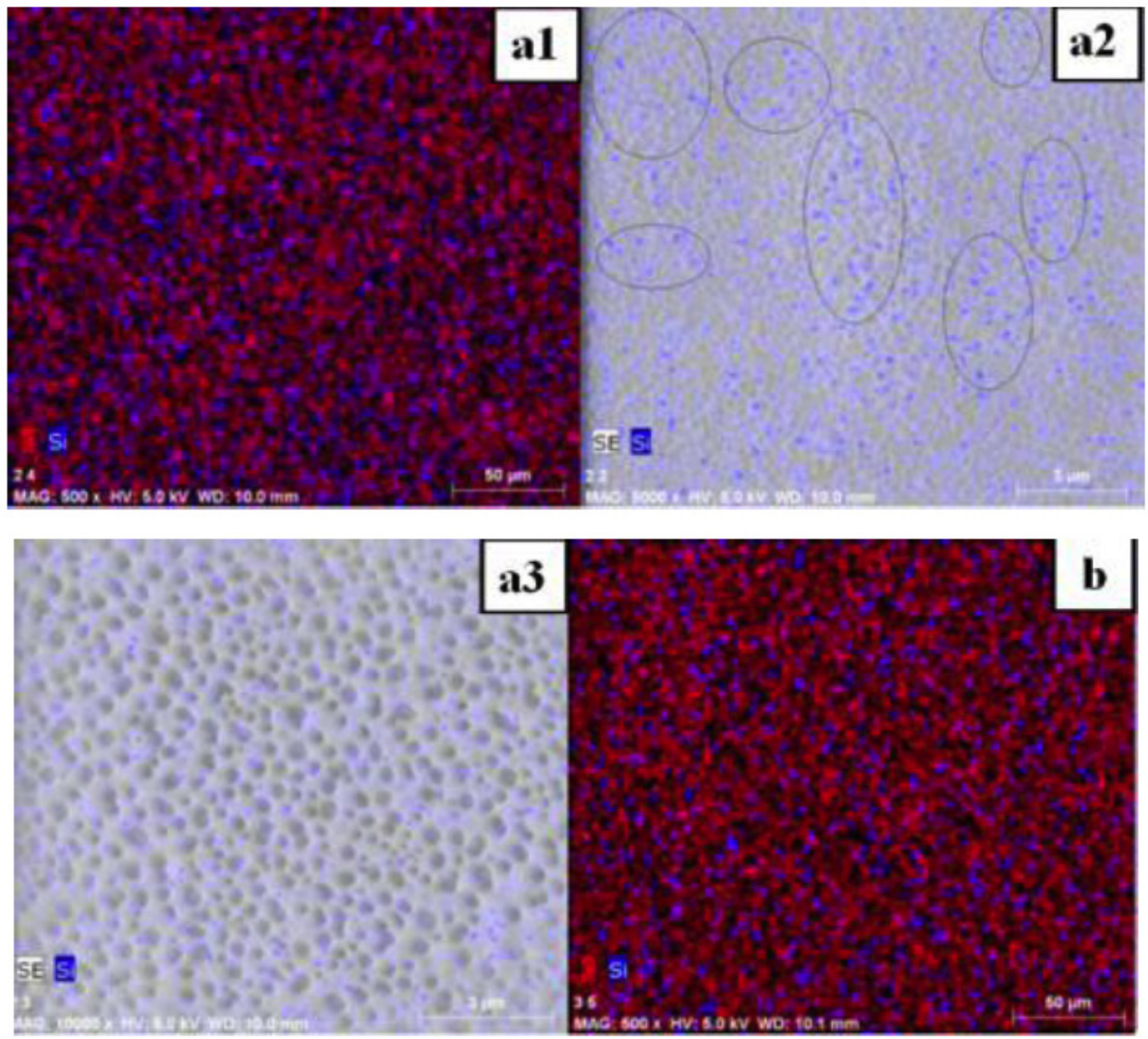

Figure 9: Element mapping on PVA stabilized PVAc dispersion film. Carbon - red; silicon - blue. VAc:GF51 weight ratio 100:3, magnification: a1 - ×500; a2 - ×5000; a3 - ×10000. VAc:GF31 weight ratio 100:1, magnification: $\mathbf{b}-\times 500$.

Surface roughness of silane modified dispersion films could impact silicon content determination. Therefore, surface structure differences between unmodified and silane modified dispersion films were evaluated by SEM (Figure 8).

According to SEM micrographs, uniform surface structure and good particle coalescence had samples prepared from PVA or surfactant stabilized unmodified PVAc dispersions (Figure 8. a, d). Application of higher VAc:Silane weight ratios in dispersion production determined increase of film surface roughness. Aggregates of polymer particles are well seen (Figure 8. $b$ and $c ; f$ and $h$ ). Neither modified nor unmodified dispersions made by RAFT method had smooth film surface. The irregular particle coalescence were determined (Figure 8. e, g, i). Film prepared from dispersion stabilized with silicon groups containing PVA (Figure 8. j) had much rougher surface, than film (Figure 8. a) cast from dispersion stabilized with silicon free PVA.

The uneven PVAc dispersion film structure could have crucial impact to silicon distribution. Impact of silicon content to film surface roughness was determined by X-Ray. The silicon mapping on dispersion films are presented in Figure $\mathbf{9}$.

According to Figure 9, uneven distribution of silicon in GF51 modified PVA stabilized dispersion film was determined. Uneven distribution could be clearly seen at both 5000 and 10000 times magnification (Figure $\mathbf{9}$. a2, a3). The given results proved presumptions about dependence of film surface roughness to silicon distribution, i.e. analytical values $5.81 \%$, theoretical $0.98 \%$ for GF51 modified PVA stabilized dispersion film (Table 1). Hence, determined theoretical value is 5.9 times lower in comparison to analytical. Generally, surface roughness of film reflected low and high concentration silicon areas.

Even though surfactant stabilized silane modified PVAc dispersion film had good silane distribution, films cast from dispersions produced by RAFT method showed opposite results. High concentration silicon areas were observed in both GF51 and GF31 modified dispersion films (Figure 10. 1, 2). It could be assumed, that silicon rich areas could be attributed to polycondensation products - oligosilanols, which were also confirmed by IR and GPC analysis. 


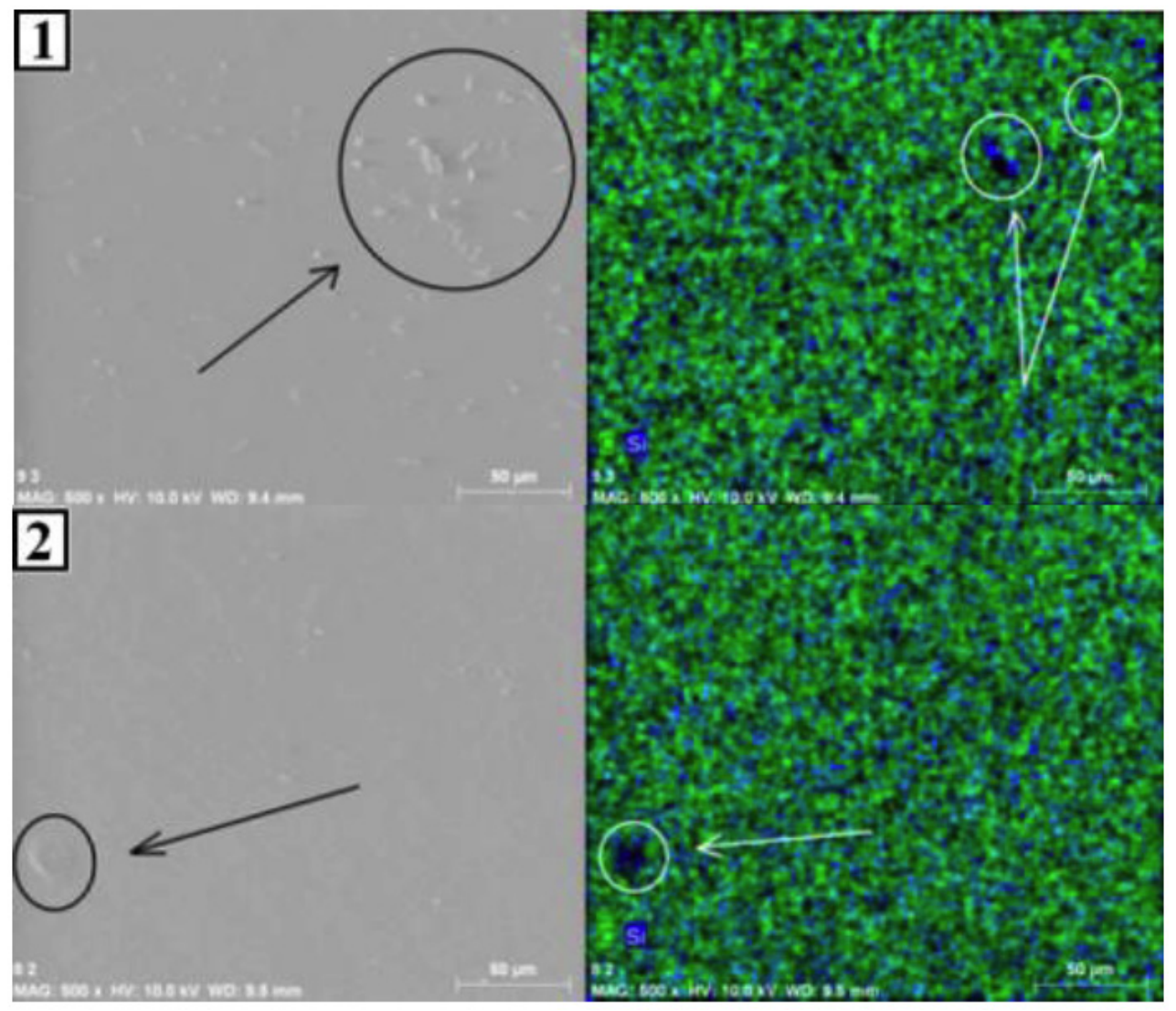

Figure 10: Element mapping on films cast from surfactant stabilized PVAc dispersions produced by RAFT method. Carbon green; silicon - blue. Magnification ×500: 1 - at VAc:GF51 weight ratio 100:3; 2 - at VAc:GF31 weight ratio $100: 1$.

\section{CONCLUSIONS}

Application of modification agents - organic silicon compounds had a great influence to polyvinyl acetate (PVAc) dispersion films structure. The macromolecular structures and bonds formed were identified by application of various modern analytical methods presented. Silanol and siloxane bond vibrations were identified by IR analysis, branched polymer structures were confirmed by application of molecular mass and weight loss analysis. The insolubility of silane modified PVAc dispersion films in various solvents completed by hydrolization procedure. Application of surfactant instead of PVA as dispersion system stabilizer, increased solubility of silane modified dispersion film. Determination of non-volatile matter, silicon content and mapping by X-Ray gave clear evidence of participation of silane compounds in PVAc dispersion films.

\section{REFERENCES}

[1] Yildirim Erbil H. Vinyl Acetate Emulsion Polymerization and Copolymerization with Acrylic Monomers. New York: CRC Press 2000.

[2] Shields J. Adhesive Handbook, $3^{\text {rd }}$ Ed. London: Butterworths 1985.
Seidel A. Vinyl acetate Polymers, in Kirk-Othmer Encylopedia of Chemical Technology, 5th Ed. New York: Wiley 2007.

[4] Tonnar J, Pouget E, Lacroix-Desmazers P, Boutevin B. Synthesis of poly(vinyl acetate)-b-poly(dimethylsiloxane-bpoly(vinyl acetate) triblock copolymers by iodine transfer polymerization. Eur Polym J 2008; 44 (2): 318-328. http://dx.doi.org/10.1016/j.eurpolymj.2007.11.026

[5] Maynard DH. Synthesis of nanophase-segregated poly(viny acetate)-poly(dimethylsiloxane) and poly(vinyl acetate)poly(styrene) graft copolymers. Polymer 2001; 42(18): 75677574.

http://dx.doi.org/10.1016/S0032-3861(01)00230-0

[6] Plesich MF, Zavaglia EA, inventor. Union carbide corp., assignee. Latex Polymers of Vinylacetate and a Silane. United States patent US 3729438. 1973.

[7] Liu XH, Yi SP, Li HB, Huang C. Preparation of Poly(vinyl acetate) Modified by Triethoxyvinylsilane and Properties of Copolymeric Lattices. Iran Polym J 2007; 16(3): 207-213.

[8] Naghash HJ, Mallakpour S, Mokhtarian N. Synthesis and characterization of silicone-modified vinyl acetate-acrylic emulsion copolymers. Prog Org Coat 2006; 55(4): 375-381. http://dx.doi.org/10.1016/j.porgcoat.2006.02.001

[9] Moriya T. inventor. Kuraray Co Ltd., assignee. Production of Vinyl Acetate - Based Polymer, Production of Saponified Vinyl Acetate-Based Polymer and Resin Composition. Japan patent JP 09071620. 1997.

[10] Stark K, inventor. Wacker Polymers Systems GmbH \& Co. KG., assignee. Silane-Modified Polyvinylacetals. United States patent US 6794477. 2004.

[11] Petrie EM. Handbook of Adhesives and Sealants. New York: McGraw-Hill 2007. 
[12] Bengtsson M, Oksman K. The use of silane technology in crosslinking polyethylene/wood flour composites. Appl Sci Manu 2006; 37(5): 752-765. http://dx.doi.org/10.1016/j.compositesa.2005.06.014

[13] Chrusciel JJ, Lesniak E. Modification of Thermoplastics with Reactive Silanes and Siloxanes. In Termoplastic Elastomers Ed. El-Sonbati A. 2012. [cited 2015 Oct 2]. Available from: http://cdn.intechopen.com/pdfs/34066/InTech-

Modification_of_thermoplastics_with_reactive_silanes_and_s iloxanes.pdf

[14] Dubininkas M, Buika G. Dispersion production by semicontinuous radical vinyl acetate emulsion polymerization with silane co-monomer and characterization of final products. Chin J Polym Sci 2013; 31(2): 346-354. http://dx.doi.org/10.1007/s10118-013-1220-0

[15] Dubininkas M, Buika G, Minelga D. Impact of industrial Grade Modified PVA to Vinyl Acetate Semi-continuous Emulsion Polymerization and Properties of Final Product. Mater Sci Medz 2013; 19(1): 38-42. http://dx.doi.org/10.5755/j01.ms.19.1.3823

[16] Dubininkas M, Buika G. Production and characterization of 3Methacryloxypropyltrimethoxysilane modified polyvinyl acetate dispersion. Kem Ind 2015.
[17] Nyquist RA, Fiedler S, Streck R. Infrared study of vinylacetate, methyl acrylate and methyl methacyrlate in various solvents. Vib Spectrosc 1994; 6(3): 285-291. http://dx.doi.org/10.1016/0924-2031(93)E0064-9

[18] Launer PJ. Infrared analysis of organosilicon compounds: Spectra-Structure correlations. Silicone compounds register and Review 1987; 100-103.

[19] Abe Y, Honda Y, Gunii T. Preparation and Properties of Silicon-Containing Polymer hybrids from 3Methacryloxypropyltrimethoxysilane. Appl Organomet Chem 1998; 12(10-11): 749-753.

http://dx.doi.org/10.1002/(SICl)10990739(199810/11)12:10/11<749::AID-AOC782>3.0.CO;2-2

[20] Donescu D, Gosa K, Ciupitoiu A, Languri I. Semicontinuous Emulsion Polymerization of Vinyl Acetate. Part I. Homopolymerization with Poly-(Vinyl Alcohol) and Nonionic Coemulsifier. J Macromol Sci 1985; 22(5-7): 931-940. http://dx.doi.org/10.1080/00222338508056645

[21] Donescu D. Emulsion Polymerization of Vinyl Acetate. London: Applied Science Publishers 1981.

[22] Brandrup J, Immergut EH, Grulke EA. Polymer Handbook. $4^{\text {th }}$ Ed. New York: John Wiley \& Sons 1999. 